\section{A Perture}

orr

\section{THE PRINCIPLES OF TREATMENT OF GASTRIC ULCER IN VIEW OF RECENT WORK.:}

\author{
BY \\ CHARLES BOLTON, C.B.E., M.D., D.Sc.,
F.R.C.P., F.R.S.,
}

PHYSICIAN TO UNIVERSITY COLLEGE HOSPITAT.

I PRopose to limit my remarks upon the treatment of ulcer of the stomach to that part of the subject which deals with the effects of disordered gastric functions upon the healing of the ulcer, the nature of these disturbances, and how they may be remedied. When the functions of the stomach are conducted in a normal manner and the diet is what may be described as normal, a gastric ulcer heals readily, but this lesion increases the irritability of the stomach so that even foods which are tolerated quite easily by the normally acting organ cause certain disturbances of function which are an important factor in arresting the healing of the ulcer. If the normal responses of the stomach to the stimulus of food can be restored and maintained the majority of ulcers will heal. This is not always easy to accomplish, although the ulcer may not have passed beyond the stage at which healing is still possible, because the patient may have been the subject of chronic functional disturbance of the stomach for many years, and his nervous system may be of that unstable type which predisposes to gastric irritability or may have been reduced to this state by an insufficient amount of food and prolonged suffering. Also the symptoms indicating gastric disturbance disappear long before the ulcer has had time to heal, and the patient is only too apt to assume that he is cured with the disappearance of his symptoms and to act accordingly.

I shall first demonstrate to you the life-history of gastric ulcer in order to show that the majority of ulcers are able to heal quite well, that the ease with which they heal depends upon their age, and that many chronic ulcers are quite incapable of healing at all.

The Life-History of Gastric Elcer.

An vlcer of the stomach originates in two distinct ways: (1) as a simple acute ulcer, or (2) as a spreading acute ulcer.

\section{Simple Acute Ulcer.}

Simple acute ulcer is formed from a strictly localized Initial lesion of several kinds, which involves the mucous membrane, submucous tissue, and of ten the muscular coat, but rarely the peritoneum. When the dead portion is separating, an artery is commonly opened up and haemorrhage results, but perforation is very rare. The uleer, once formed, has no great tendency to spread except sometimes in an irregular fashion, but remains localized to the area of mucous membrane initially damaged, its size as determined by this area being small but occasionally reaching an inch in diameter.

The healing of these ulcers is usually completed in from three to four weeks. If the patient dies from haemorrhage in the meantime the new mucous membrane will be found to have grown up to the edge of the bleeding vessel, which is only to be found by careful search. Sometimes, however, the healing takes place slowly and with difficulty, being delayed for several months. Such ulcers thicken and the connective tissue base may become excavated and the healing arrested.

\section{Spreading Acute Ulcer.}

This form does not result from the mere separation of a dead portion of the wall of the stomach like the preceding type, but begins at one spot in the mucous membrane and spreads in a circular or oval fashion, destroy\footnotetext{
* Delivered before the Guildford Division of the British Medical
Association, December 13th, 1923.
}

ing one layer of the wall after another and giving rise to a terraced edge to the ulcer. It forms a rounded or oval-ulcer like a small spoon, and later, when the muscular coat is destroyed, the terracing of the edge disappears and the base becomes thickened and raised. Large shallow ulcers are so formed, and the peritoneal base becomes adherent to the liver, pancreas, or omentum; otherwise it thickens and resists perforation. The ulcer tends to bleed, and may perforate at the edge, which becomes undermined by sloughing of the newly formed connective tissue. It may, on the other hand, penetrate the coats of the stomach in the shape of a funnel and perforate at the apex (acute perforating ulcer). These spreading ulcers heal with surprisingly little thickening to be seen, or they may become chronic.

\section{Chronic Ulcer.}

Chronic ulcer may arise in two ways:

(1) By the failure to heal of a simple acute ulcer a chronic ulcer is formed, which is usually small but occasionally of moderate size, and rounded or oval in shape with a thickened base sometimes excarated into a rounded cavity.

(2) By the failure to heal of a spreading acute ulcer two types of chronic ulcer are produced : (a) The first is a large flat ulcer which has a thickened base, or the base has disappeared, exposing the liver, pancreas, or omentum, which thus come to form part of the wall of the stomach. (b) The second type has not laid bare any structure, but its base has become very thick, sometimes enormously so. Its cavity may be funnel-shaped, with sometimes a perforation at the apex, or it may be excavated into a globular or irregular hollow. Such ulcers not uncommonly form palpable tumours.

Many of these ulcers are capable of healing if the edges can be brought reasonably close together and a cellular stroma is able to cover the base, but the interposition of an exposed organ or a deep cavity not uncommonly offers a permanent obstacle to healing. All ulcers may thus heal unless they have arrived at this very advanced condition, and they heal with greater ease in the earlier stages of their development.

This is the life-history of gastric ulcer as I read it, and all that $I$ have observed since $I$ published my first communication on the origin of chronic ulcer from the acute variety has tended to confirm this yiew.

The Effects of Ulcer upon the Gastric Functions.

The ulcer acts as an irritant and affects the neuromuscular mechanism of the stomach, the acidity of the gastric contents, and the amount of gastric juice secreted.

The pyloric region is the most important part affected. (1) Irritability of the pyloric sphincter is produced and its function as the regulator of the output of the stomach is interfered with. It fails to relax in its normal fashion, and finally becomes spasmodic. Delay in the emptying of the stomach results. (2) A second function of the pylorus is to regulate the acidity of the gastric contents. As the secreted gastric juice mixes with the food the percentage of combined and free $\mathrm{HCl}$ in the gastric contents gradually rises to about $0: 2$, when increased relaxation of the pylorus occurs and allows of the regurgitation of bile and pancreatic juice into the stomach,- which neutralizes the HCl with the formation of inorganic chloride, and the acidity falls as the stomach empties. This function is also interfered with by the failure of the pylorus to relax, and the percentage of $\mathrm{HCl}$ continues to rise and maintains its height to varying degrees as the stomach empties. Hyperchlorhydria is thus produced. (3) The prolongation of the digestive process gives rise to hypersecretion of the gastric juice in many cases. These three results become permanent when organic pyloric obstruction supervenes.

An ulcer of the body of the stomach causes a ring spasm at the spot, but the acidity of the gastric conteints is not interfered with unless the pylorus becomes irritable or hypersecretion is present. When a tight hour-glass constriction results hypersecretion is set $u$ p in the proximal sae, and neutralization of the contents of this sac is impeded. 
The Effects of these Disorders on the Healing OF ULCER.

There is only one way by which to approach this problem, and that is by the experimental method. I will now give you a demonstration of the results of my observations upon the subject.

There are four stages in the healing of a gastric ulcer, which, of course, overlap: (1) The necrotic tissue and sloughs become separated from the base. (2) The base becomes covered with healthy granulation tissue. (3) The epithelium at the margin of the ulcer grows over the base in a single layer of flat cells which become cubical. Glands, beginning as invaginations in the covering epithelium, gradually grow and burrow in the cellular stroma, forming a new mucous membrane. In the meanwhile the edges of the ulcer approach one another by contraction of the fibrous tissue formed in the deeper layers of the base. The area thus left to be covered by epithelium varies somewhat in size in different cases, but this makes very little difference in the actual time of healing if the base of the ulcer is clean.

Effects of Variations in the Diet of the Animal.

Differences in diet give the stomach different degrees of work with regard to secretion and motion. The cat is a suitable animal for such experiments, because after a full meal of milk the stomach is empty in about three hours, whereas after a full meal of meat it is not empty for about twelve hours. In a series of experiments, in which gastrotoxic ulcers were produced in these animals, I found that the base of the ulcer in milk-fed cats was in general completely covered with epithelial cells on the twentieth day, but that in the meat-fed cats the base was entirely uncovered, or the epithelium had reached the periphery but had left a large uncovered area in the centre of the ulcer. This delay in growth of the epithelium was due to necrosis of the superficial cells of the granulation tissue base of the ulcer offering an effectual barrier to the overgrowth of the epithelium. This necrosis was due to the prolonged action of the $\mathrm{HCl}$ of the gastric juice, and was accompanied by excessive formation of fibrous tissue in the base of the ulcer. The meat remained longer in the stomach than the milk and excited a more copious flow of gastric juice, with the result that the ulcer was delayed in healing owing to necrosis of its base, the period of delay, however, not exceeding a variable number of days.

Effects of Delay in the Emptying of the Stomach.

Delay in the emptying of the stomach beyond the normal time and dilatation of the organ were produced by artificial pyloric stenosis in the cat. In a series of experiments I found that the healing of ulcers produced in these animals was delayed in proportion to the degree of retention of the food in the stomach. The delay occurred in the early stages and was due to necrosis of the granulation tissue covering the base of the ulcer, the epithelium being unable to grow over it. In one case at the end of six weeks the centre of a thick sclerotic base was uncovered. This fibrous condition of the base provides a very unfavourable stroma for the regenerated gastric glands to proliferate in, and the newly formed mucous membrane consisted in these cases of glands of the duct type only, dilated and of very irregular shape. These changes were due not only to prolongation of the digestive process, but also to deficient neutralization of the $\mathrm{HCl}$ in the gastric contents by interference with the normal duodenal regurgitation. The effects in these and the succeeding experiments were naturally very much more marked than in those dealing with mere variations in diet, the period of delay extending to weeks.

\section{Effects of Administration of $\mathrm{HCl}$.}

In these experiments monkeys were used, the $\mathrm{HCl}$ being administered by tube early in the morning. The acidity falls rapidly from neutralization by duodenal regurgitation of pancreatic juice, so that the HCl has only a short time in which to act. Using strengths of 0.18 per cent. $\mathrm{HCl}$ and upwards, a pronounced delay of two or three times the normal period of healing was observed, due to necrosis of the base of the ulcer as described above. In such cases the newly formed mucous membrane was of the atypical variety. Strengths of 0.1 per cent. $\mathrm{HCl}$ and below produced no appreciable effect. Matthes had already found that 0.5 per cent. solution of $\mathrm{HCl}$ delayed the healing of an ulcer in a dog. This method is a very imperfect imitation of hypersecretion and hyperchlorhydria.

\section{Administration of $\mathrm{HCl}$ together with Pyloric Obstruction.}

Monkeys were again used and the degree of obstruction confirmed by the $x$ rays. In these cases not only was hyperacidity of the contents and delayed emptying of the stomach secured, but duodenal regurgitation and neutralization were interfered with. A summation of effects was here observed. In all cases on the twenty-first day the base of the ulcer was covered with a large thick slough, which effectively prevented the epithelium from growing over: the base.

In human pathology precisely the same condition of the base is seen in acute ulcers which are delayed in their healing, and in chronic ulcers the healing of which is arrested-namely, necrosis of the connective tissue and secondary inflammatory thickening-and this condition of the base is the reason why the ulcers are delayed or arrested in their healing. Delayed emptying of the stomach with prolongation of the digestive process, interference with the normal duodenal regurgitation which regulates the acidity of the gastric contents, and hypersecretion of the gastric juice, are the functional disturbances of the stomach which play their part in bringing about this state of affairs.

\section{Treatment.}

Restoration of the Normal Gastric Functions.

The two objects we have in view are: (1) to lessen the neuro-muscular irritability of the stomach, more particularly of the pylorus, and thereby facilitate the emptying of the organ and restore the normal degree of duodenal regurgitation; and (2) to reduce the amount of gastric juice secreted. It is important to recognize at the outset that the disappearance of pain is no index of the restoration of normal gastric function and still less of the healing of the ulcer: The food may be held up by the pylorus without exciting pain, and hypersecretion may also be present without the existence of this symptom. Crohn has shown that hypertension of the pylorus is much more easily overcome than hypersecretion, which requires prolonged treatment, although pain has been long absent. Tlre degree of pyloric tone and the amount of gastric juice secreted vary in different people, and our aim is to restore our patient to the condition which is normal for him as an individual. The age and condition of the ulcer can only be surmised in a vague kind of way, and since a chronic ulcer takes many months to heal we must divide the treatment devised to accomplish the two objects mentioned above into two stages: (1) preliminary treatment, and (2) the subsequent treatment.

Preliminary treatment lasts six weeks, during which the patient is put to bed to reduce his expenditure of energy, so that the diet may be reduced to a minimum, thus diminishing the stimulus of food and the work of the stomach. The excessive irritability rapidly disappears and the work of the stomach is then gradually increased by increase of food until the patient is eating the full diet he is to be allowed. He then gets up and proceeds to the subsequent treatment, during which he eats the full diet allowed-otherwise malnutrition and debility of the nervous system will result-masticating his food thoroughly and taking sufficient time and rest with his meals.

Diet.

In order to reduce the irritability of the pylorus the food in the preliminary stage must be liquid or semi-solid, finely divided, and free from irritants. The gastric contents must be neutralized by an alkali during the later stages of digestion as the $\mathrm{HCl}$ acts as an irritant to the pylorus. In the subsequent treatment the food must be such as to be easily disintegrated and peptonized, and free from irritants 
such as decomposed fats, acids, and food accessories, otherwise irritability of the pylorus will be excited.

In order to reduce the amount of gastric juice secreted food must be selected which not only leaves the stomach easily, but which gives rise to a moderate flow only of this secretion. Here I will remind you of one or two fundamental points with regard to the amount of gastric juice secreted in response to the ingestion of different protein foodstuffs. The extractives contained in meat, and therefore in soups, beef-tea, and meat extracts, give rise to a maximal flow of gastric juice and in comparison they contain very little protein to take up the acid. The flow is especially large if they are retained longer in the stomach by admixture with an inert substance such as starch. This is the reason that meat causes such a large flow unless the extractives are boiled out of it. Beef and mutton are more difficult to disintegrate than chicken and fish, stay longer in the stomach, and hence excite a greater flow of gastric juice. Bread causes a much smaller secretion, but it is liable to be prolonged. All vegetable proteins are more difficult to digest than animal proteins. Milk also causes a smaller secretion than meat, and leaves the stomach easily unless tough clots are formed; but this can be overcome by citrating. Raw egg causes a minimum or no secretion at all; if boiled, more juice is excited. Fats inhibit secretion and lower its digestive power.

In the preliminary treatment the foods are therefore presented in the following order, during the six weeks in bed: milk, raw eggs, thin bread-and-butter, boiled eggs, cereals boiled in milk, pounded fish or chicken, mashed potato, sieved green vegetables, ordinary fish, chicken, mutton. Meat extracts are not given at all, nor meats nor other foods which are not easily disintegrated. The feeds should be given every three hours, beginning with $7 \mathrm{oz}$. of milk at 7 a.m., 10 a.m., 1 p.m., 4 p.m., 7 p.m., 10 p.m., and afterwards three meals a day are built up in the above stages at 7 a.m., 1 p.m., and 7 p.m., or other convenient hours. These are the principles, and the details should be arorked out for individual cases by the practitioner in attendance. In general the food should pass the prlorus easily and excite the secretion of gastric juice as little as possible. The subsequent diet allowed should contain an adequate amount of protein and carbohydrate, and as much fat as the patient likes in the form of butter, cream, boiled bacon, and oil if he chooses.

The idea of giving an excess of fat in the form of olive oil in order to inhibit the secretion of gastric juice was suggested by Cohnheim. Such a diet may be encouraged in the case of people who like and can tolerate it, but they are in the minority. The oil is apt to excite nausea and eructations, and even diarrhoea and vomiting in many people. We must also remember that an excess of fat may cause an after-secretion of gastric juice.

An excess of protein forms the basis of the diet in the Lenhartz treatment. The first idea is that the excess of protein combines with the free acid of the gastric juice which would otherwise remain in the free state and damage the ulcer. The feeds are not given at stated times but in small quantities very frequently during the day, ithd consist in the first instance of eggs beaten up with sugar and milk. The amount is increased each day, the eggs are boiled and other foods are added, so that at the end of a fortnight the daily ration would be eight eggs, a pint and a half of milk, two ounces of minced meat, boiled rice, and bread-and-butter. The eggs are then gradually reduced and more meat added to replace them, and other foodstuffs up to a full mixed diet, the patient getting up at the end of a month. The second idea is that anaemia and malnutrition prevent the healing of the ulcer, and therefore the above treatment is commenced at once whether bleeding is taking place or not.

My criticisms of the Lenhartz diet are: (1) That it is a wrong principle to endeavour to neutralize all the free acid by giving excess of protein, which simply increases the secretion according to the amount given and the time it remains in the stomach. Such a diet is particularly liable to increase any hypersecretion which may be present. (2) That it is much better to give meals at stated times such as the patient has been accustomed to, and to neutralize the $\mathrm{HCl}$ by alkalis about one and a half to two hours after each meal, as occurs naturally. (3) That it is wrong to feed a bleeding patient, because the blood clot which is closing the artery is easily dislodged by the movements of the stomach and by the vasomotor dilatation consequent on the ingestion of food, and it is disintegrated by the gastric juice. Loss of blood is no obstacle to the healing of the ulcer, as I have shown you at the beginning.

Rectal feeding should only be resorted to under two conditions: (1) bleeding from the stomach; (2) uncontrollable vomiting, which is an uncommon complication of gastric ulcer. The period of feeding need never be prolonged beyond a few days, and as soon as possible small mouth feeds are given frequently and the intervals gradually lengthened to three hours.

\section{Administration of Alkalis.}

We have seen that during digestion when the acidity due to $\mathrm{HCl}$ has risen to the average height of about 0.2 per cent. the pylorus relaxes and regurgitation of pancreatic juice occurs into the stomach and brings down the acidity. When the stomach is in the fasting condition and gastric juice is being secreted in normal or abnormal quantity the pylorus is continually relaxing to let out this fluid and permit of its neutralization by regurgitation of the intestinal juices. In the majority of patients with gastric ulcer this pyloric relaxation and neutralization of the gastric contents is deficient. The correct procedure to adopt in order to replace this deficiency is to give alkalis about one and a half to two hours after each meal, and also once or twice during the night if the patient happens to be awake. By doing this the gastric contents are neutralized in the normal way and the hypersecretion is gradually reduced. The best alkalis to administer are bismuth carbonate, calcium carbonate, magnesium oxide, and sodium bicarbonate. Sodium carbonate is the chief neutralizer in the normal condition.

This treatment by alkalis has been unnecessarily carried to excess by Sippy. His method aims at completely neutralizing the $\mathrm{HCl}$ in the gastric contents. Food is given every hour from 7 a.m. to 7 p.m., and alkalis are also given every hour between the feeds and also every halfhour between 7 p.m. and 10 p.m. The doses of alkalis vary, and sometimes large doses are used. My criticisms of this method are: (1) That the gastric juice is put out of action and digestion stopped. (2) That this amount of neutralization is quite unnecessary, since free $\mathrm{HCl}$ below 0.1 per cent. does no material damage, certainly not sufficient to delay the healing of an ulcer. (3) According to Hardt and Rivers this excessive and constant giving of alkalis in some people produces symptoms of intoxication.

We must remember that we are not dealing with a mixture of chemical substances in a test tube, but that we are treating a human being, and the best results will be obtained by putting him in the most natural condition pessible.

\section{Atropine.}

This drug has its uses, but takes a distinctly secondary place in the treatment. It varies very much in its effects in different cases, and, in general, to produce a definite result large doses are required, so that dryness of the mouth and other adverse symptoms are liable to result. Its most important action in reducing the acidity of the gastric contents lies in its power to relax the pyloric sphincter and permit of duodenal regurgitation, the actual diminution in the amount of secretion during digestion being of much less account. The hypersecretion in the fasting stomach can be stopped by atropine, as shown by Crohn, if large doses are administered.

\section{Surgical Treatment.}

The only direct means of dealing with a chronic ulcer is to excise it. Otherwise the aim of surgery is precisely the same as that of medicine-namely, (1) to facilitate the emptying of the stomach, and (2) to induce the regurgitation of intestinal fluids into the stomach, thereby neutralizing its contents in imitation of the normal duodenal regurgitation. I cannot, on this occasion, discuss the prophylactic and other aspects of the treatment. 